\title{
ON THE DEGREE OF CONVERGENCE OF EXTREMAL POLYNOMIALS AND OTHER EXTREMAL FUNCTIONS( ${ }^{(1)}$
}

\author{
BY \\ J. L. WALSH AND A. SINCLAIR
}

1. Introduction. This paper is motivated by the following problem [19, $\S 11.3]$. Let a given function $F(z)$ be of class $L^{p}, p>1$, on an analytic Jordan curve $\gamma$ in the plane of the complex variable $z$, and let $p_{n}(z)$ be the unique sequence of polynomials in $z$ of respective degrees $n$ of best approximation to $F(z)$ on $\gamma$ in the sense of minimizing $\int_{\gamma}\left|F(z)-p_{n}(z)\right|^{p}|d z|$; these minimizing $p_{n}(z)$ may also be subjected to certain auxiliary conditions of interpolation $p_{n}\left(w_{k}\right)=u_{k}, k=1,2, \cdots, m$, which are independent of $n$ and are not necessarily related to $F(z)$. The object is to study convergence and degree of convergence of the sequence $p_{n}(z)$ to a possible limit minimizing function and to study various properties of this limit function. We attack this problem first (Part I) by studying a general situation in $L^{p}$-space, and then (Part II) by specializing to the problem already mentioned. We treat likewise the analogous problem where best approximation is measured by a surface integral over the interior $C$ of $\gamma$.

Related problems for special cases have been studied by Bieberbach [2], Julia [7], Keldyš and Lavrentieff [9], and Smirnoff [17]. For an analytic Jordan curve approximation in the case of arbitrary $m$ and $p=2$ was studied by Walsh $[19, \$ 11.5]$, including complete determination of the limit function in closed form and (where appropriate) maximal convergence of the extremal polynomials. The corresponding problem of approximation, including maximal convergence, with $m=1$ and $p$ arbitrary was considered by Spitzbart [18]. Approximation by functions analytic and bounded in a region $D$ containing $\bar{C}$ was treated by Walsh and Russell [23], especially in the case $p=2$. The minimizing functions have been studied by Kakeya [8], Doob [4], Penez [11], Macintyre and Rogosinski [10], and Rogosinski and Shapiro [13]. F. Riesz and M. Riesz [12] studied the boundary values of an analytic function in $H_{p}$. A paper by Rosenbloom and Warschawski [14] contains in outline some material related to work in the present paper, without detailed results; the present research was commenced and completed without use of that material.

\footnotetext{
Presented to the Society, August 27, 1958; received by the editors March 23, 1960 and, in revised form, June 4, 1963.

$\left({ }^{1}\right)$ This research was supported (in part) by the United States Air Force Office of Scientific Research, Air Research and Development Command, and (in part) by the Office of Naval Research.
} 
For $p \neq 2$ we rely on suitable inequalities and make large use of the orthogonality condition (Theorem 4) which characterizes the extremal function $f^{*}$. Continuity properties of the extremal function enable us to estimate $\left\|p_{n}\right\|-\left\|f^{*}\right\|$, and inequalities depending on the uniform convexity of $L^{p}$ to estimate $\left\|f_{n}^{*}-p_{n}^{*}\right\|$. Conclusions are obtained involving degree of convergence, and overconvergence, i.e., convergence in a larger region. (Compare with the simpler case, $p=2[19, \S 11.5$, Theorem 9].)

In Part I we are concerned with a closed convex subset $L_{*}$ of $L^{p}$ with closure by the $L^{p}$-norm topology. If $f(z)$ is analytic interior to the unit circle and if the integrals $\int_{0}^{2 \pi}\left|f\left(r e^{i \theta}\right)\right|^{p} d \theta, 0<r<1$, are bounded, $f(z)$ is said to be of class $H_{p}$ and it is well known that for radial approach or approach "in angle" has boundary values almost everywhere on the unit circle and that for these boundary values the integral $\int_{0}^{2 \pi}\left|f\left(e^{i \theta}\right)\right|^{p} d \theta$ exists. The subclass $H_{p}$ of $L^{p}$ on $|z|=1$ so determined is known to be a closed subset of $L^{p}$. For the case that $\gamma$ is an analytic Jordan curve, $H_{p}(\gamma)$ is defined in the obvious way by a conformal mapping onto the interior of the unit circle. The closure on $\gamma$ of $H_{p}$ in $L^{p}$ is evident.

If $L^{p}$ refers to the surface integral taken with respect to area over $C$, a limited region of the complex plane, $H_{p}^{\prime}$ is defined as the subset of functions of $L^{p}$ analytic on $C$. If $f_{m}, f_{n} \in H_{p}^{\prime}$ and $\lim _{m, n \rightarrow \infty} \iint_{C}\left|f_{n}-f_{m}\right|^{p} d S=0$, there exists $f \in L^{p}$ such that $\lim _{n \rightarrow \infty} \iint_{C}\left|f-f_{n}\right|^{p} d S=0$. Then $\lim _{n \rightarrow \infty} f_{n}(z)=f(z)$ uniformly on any closed set interior to $C\left[19\right.$, p. 109]. Thus $H_{p}^{\prime}$ is closed.

A subset $L_{*}$ of $L^{p}$ is called a convex subset of $L^{p}$ provided that $g, h \in L_{*}$ implies $[r g+(1-r) h] \in L^{*}$ for all $r$ such that $0 \leqq r \leqq 1$. We say $L_{n}$ is a nested sequence of subsets of $L^{p}$ if $L_{n} \subset L_{n+1} \subset L^{p}, n=1,2, \ldots$ In the applications considered in this paper (Part II) $L_{*}$ is the closure of $\bigcup_{1}^{\infty} L_{n}$.

It is assumed throughout this paper that $C$ is a limited region of the complex plane and that integration is over $E$, namely, the boundary $\gamma$ of $C$ or the point set $C$ itself. In the former case we suppose $\gamma$ is an analytic Jordan curve and integrate with respect to arc length; in the latter case we integrate with respect to area. If $\int_{E}\left|f_{1}-f_{2}\right|^{p} d \mu=0, f_{1}$ and $f_{2}$ are regarded as the same function on $E$.

A polynomial $p_{n}(z)$ of degree $n$ is a function which can be expressed in the form $a_{0} z^{n}+a_{1} z^{n-1}+\cdots+a_{n}$, where $a_{0}$ may be zero. We give examples for later reference of closed convex subsets $L_{*}$ of $L^{p}$ and nested sequences $L_{n}$ of such sets.

Example A. For $L_{*}=H_{p}$ (or $H_{p}^{\prime}$ ) and $L_{n}$, the set of polynomials of degree $n, L_{n}$ is a nested sequence of closed convex subsets of the closed convex subset $L_{*}$.

Example B. Given points $w_{1}, w_{2}, \cdots, w_{m}$ interior to $C$, let interpolation conditions $f\left(w_{j}\right)=u_{j}, j=1,2, \cdots, m$, be assigned. If the $w_{j}$ are not all distinct, the appropriate $u_{j}$ are to be interpreted in the usual way as values 
assigned to derivatives. Define $L_{*}=\left\{f \mid\right.$ (1) $f \in H_{p}$ (or $\left.H_{p}^{\prime}\right) ;$ (2) $f\left(w_{j}\right)=u_{j}$, $j=1, \cdots, m\}$ and $L_{n}=\left\{f \mid(1) f \in L_{*} ;(2) f(z)=p_{n}(z)\right\}$. It is known that the function $f_{p}^{*}$ of $L_{*}$ which minimizes $\int_{|z|=1}|f(z) .|^{p}|d z|$ is of the form

$$
A \prod_{i \in S^{\prime}}\left(z-\alpha_{i}\right) /\left(1-\overline{\alpha_{i}} z\right) \prod_{i \in S}\left(1-\overline{\alpha_{i}} z\right)^{2 / p} \prod_{j=1}^{m}\left(1-\bar{w}_{j} z\right)^{-2 / p},
$$

where $\left|\alpha_{i}\right|<1, S$ is the set of integers $1,2, \cdots, m-1$, and $S^{\prime}$ is a certain subset of $S[10$, pp. 277-278].

\section{RESULTS FOR A GENERAL $L^{p}$-SPACE}

2. Existence of extremal function. It is well known that if $L_{*}$ is a closed convex subset of $L^{p}, 1<p<\infty$, there is a unique element of $L_{*}$ for which $\|g\|_{p}=\left\{\int_{E}|g|^{p} d_{\mu}\right\}^{1 / p}$ is a minimum $[1$, p. 103], [25, p. 129]. We assume that the measure $\mu$ of $E$ is finite. In this paper extremal function of $L_{*}$ denotes a function $g^{*}$ which minimizes $\|g\|_{p}, g \in L_{*}$.

Theorem 1. If $F \in L^{p}$ and $L_{*}$ is a closed convex subset of $L^{p}$, then

$$
L_{*}^{\prime}=\left\{g \mid g=F-f, f \in L_{*}\right\}
$$

is also a closed convex subset of $L^{p}$. There exists a unique element $f^{*}$ of $L_{*}$ for which $\|F-f\|_{p}$ is a minimum, namely: $f^{*} \equiv F-g^{*}$, where $g^{*}$ is the extremal function of $L_{*}^{\prime}$.

In Theorem 2 the weight function $w$ is understood to be positive, integrable, and bounded from zero on $E$.

THEOREM 2. If $L_{*}$ is a closed convex subset of $L_{w}^{p}$ (the set of functions $f$ such that $\int_{E} w|f|^{p} d_{\mu}$ exists) when $\left[\int_{E} w|f|^{p} d_{\mu}\right]^{1 / p}$ is taken as $\|f\|_{p}$, then

$$
L_{*}^{\prime}=\left\{g \mid g=w^{1 / p} f, f \in L_{*}\right\}
$$

is a closed convex subset of $L^{p}$. Hence, there exists a unique element $f^{*} \in L_{*}$ for which $\|f\|_{p}$ is a minimum, namely: $f^{*}=g^{*} /(w)^{1 / p}$, where $g^{*}$ is the extremal function of $L_{*}^{\prime}$.

By combining the results of Theorems 1 and 2 we obtain the following: If $w$ is a weight function as described above and if $F$ is an arbitrary element of $L_{w}^{p}$ then for $f$ in $L_{*}$, a closed convex subset of $L_{w}^{p}$, there is a unique $f^{*}$ of $L_{*}$ such that $\int w|F-f|^{p} d_{\mu}$ is a minimum and $f^{*}=F-g^{*} /(w)^{1 / p}$.

3. Inequalities for norms in an arbitrary $L^{p}$-space. As is customary,

$$
\left\{\int_{E}|g|^{p} d \mu\right\}^{1 / p}
$$

is abbreviated by $\|g\|_{p}$ or just by $\|g\|$. 
Lemma 3.1. Suppose $p>0$ and choose $r, 0<r<1$. Then, for $|Z|<r<1$, $|1+Z|^{p}=1+p \operatorname{Re} Z+R(Z)$ with $|R(Z)|<|Z|^{2} A(p, r)$ with $A(p, r) d e-$ pendent on $p$ and $r$ but independent of $Z$.

Proof. We have

$$
(1+Z)^{p / 2}=1+(p / 2) Z+M(Z) \quad \text { with }|M(Z)|<K(p, r)|Z|^{2} .
$$

Then

$$
\begin{aligned}
|1+Z|^{p} & =[1+(p / 2) Z+M(Z)][1+(p / 2) \bar{Z}+\bar{M}(Z)] \\
& =1+p \operatorname{Re} Z+R(Z), \quad \text { as required. }
\end{aligned}
$$

The method of proof used for Theorem 3 is similar to one used by Ahlfors [1, pp. 121-122].

Theorem 3. Choose $r, 0<r<1$. Suppose $p>1$ and $f, g \in L^{p}$ with integration over $E$. Let $E_{1}$ denote the subset of $E$ on which $|g / f|<r$ and $E_{2}$ the subset of $E$ on which $|g / f| \geqq r$. Then

(a) $\quad \int_{E_{1}}|f+g|^{p} d \mu=\int_{E_{1}}|f|^{p} d \mu+p \operatorname{Re} \int_{E_{1}}|f|^{p}(g / f) d \mu+\int_{E_{1}} R_{1} d \mu$,

$$
\left|R_{1}(X)\right|<|f(X)|^{p}|g(X) / f(X)|^{2} A(p, r) ;
$$

(b) $\int_{E_{2}}|f+g|^{p} d \mu=\int_{E_{2}}|f|^{p} d \mu+p \operatorname{Re} \int_{E_{2}}|f|^{p}(g / f) d \mu+\int_{E_{2}} R_{2} d \mu$,

$$
\left|R_{2}(X)\right| \leqq|g(X)|^{p} B(p, r) .
$$

Proof. On $E_{1}$ Lemma 3.1 implies

$$
|f|^{p}|1+(g / f)|^{p}=|f|^{p}[1+p \operatorname{Re}(g / f)+R(g / f)] .
$$

The result (a) is immediate.

Since on $E_{2}$ we have $|g / f| \geqq r$,

$$
|| f+\left.g\right|^{p}-|f|^{p}-\left.p \operatorname{Re}|f|^{p}(g / f)|\leqq| g\right|^{p}\left[(1+1 / r)^{p}+(1 / r)^{p}+p(1 / r)^{p-1}\right] .
$$

Inequality (b) follows directly.

A subset $L_{*}$ of $L^{p}$ with an extremal element $f$ will be called admissible if for some $b$, which may depend on $h, 0<b \leqq \infty$, the relation $h \in L_{*}$ implies $f+\lambda(f-h) \in L_{*}$ for $\lambda$ complex and $|\lambda| \leqq b$.

THEOREM 4. Suppose $f$ is extremal for an admissible subset $L_{*}$ of $L^{p}$ and let $h$ be any element of $L_{*}$. Then

$$
\int_{E}|f|^{p}(f-h) / f d \mu=0 .
$$


Proof. In Theorem 3 set $g=\lambda \phi$ with $\phi \equiv f-h$ and denote $E_{1}$ and $E_{2}$, which now depend on $\lambda$, by $E_{1_{\lambda}}$ and $E_{2_{\lambda}}$. Addition of (a) and (b) yields:

$$
\begin{aligned}
\int_{E}|f+\lambda \phi|^{p} d \mu= & \int_{E}|f|^{p} d \mu+p \operatorname{Re} \int_{E}|f|^{p}(\lambda \phi / f) d \mu \\
& +\int_{E_{1_{\lambda}}} R_{1} d \mu+\int_{E_{\lambda_{\lambda}}} R_{2} d \mu
\end{aligned}
$$

$$
\left|R_{1}(X)\right|<|\lambda|^{2}|f(X)|^{p-2}|\phi(X)|^{2} A(p, r), \quad\left|R_{2}(X)\right| \leqq|\lambda|^{p}|\phi(X)|^{p} B(p, r) .
$$

For $p \geqq 2$, it follows from the Hölder inequality and the fact the integral is not decreased by substituting $E$ for $E_{1}$ that the third term on the right is bounded by $A(p, r)\|f\|_{p}^{p-2}|\lambda|^{2}\|\phi\|_{p}^{2}$. Clearly the fourth term on the right side of (4.2) is bounded by $B(p, r)|\lambda|^{p}\|\phi\|_{p}^{p}$. For $1<p<2$, the third term on the right side of (4.2) is bounded by $A(p, r)|\lambda|^{2} \int_{E}|f|^{p}|\phi / f|^{p} d \mu$, that is, by $A(p, r)|\lambda|^{2}\|\phi\|_{p}^{p}$, and the fourth term by $B(p, r)|\lambda|^{p}\|\phi\|_{p}^{p}$.

Since $f$ is extremal for $L_{*}$ and $(f+\lambda \phi) \in L_{*}$ for $|\lambda| \leqq b$, we have

$$
\|f+\lambda \phi\|^{p} \geqq\|f\|^{p},
$$

whence

$$
0 \leqq p \operatorname{Re} \int \lambda|f|^{p-2} \bar{f} \phi d \mu+O\left(|\lambda|^{s}\right), \quad \lambda \rightarrow 0,
$$

with $S=2$ for $p \geqq 2, S=p$ for $1<p<2$. If (4.1) is not satisfied, $\arg \lambda$ can be assigned so that the argument of the first term on the right side of (4.3) is $\pi$. For $|\lambda|$ sufficiently small the modulus of the first term exceeds that of the second; this gives a contradiction and completes the proof. For this proof, compare [5a].

THEOREM 5. Suppose $f$ is extremal for an admissible subset $L_{*}$ of $L^{p}$ and that $\pi_{n}$ is a sequence in $L_{*}$ such that $\left\|f-\pi_{n}\right\|_{p}<1$. We conclude the following:

(a) $\left\|\pi_{n}\right\|_{p}^{p}-\|f\|_{p}^{p}<M_{1}\left\|f-\pi_{n}\right\|_{p}^{2}$ for $p \geqq 2$.

(b) If $f$ is bounded on $E$ and if $\left|f(X)-\pi_{n}(X)\right|<1$ almost everywhere on $E$, then

$$
\left\|\pi_{n}\right\|_{p}^{p}-\|f\|_{p}^{p}<M_{2}\left\|f-\pi_{n}\right\|_{2}^{2} \text { for } p \geqq 2 ;
$$

if $|f|^{p-2}$ is bounded on $E$ and if $\left|\left(f(X)-\pi_{n}(X)\right) / f(X)\right|<r<1$ almost everywhere on $E$, then

$$
\left\|\pi_{n}\right\|_{p}^{p}-\|f\|_{p}^{p}<M_{3}\left\|f-\pi_{n}\right\|_{2}^{2} \text { for } 1<p<2 .
$$

(c) If $\left|\left(f(X)-\pi_{n}(X)\right) / f(X)\right|$ is bounded on $E$, then

$$
\left\|\pi_{n}\right\|_{p}^{p}-\|f\|_{p}^{p} \leqq M_{4}\left\|f-\pi_{n}\right\|_{p}^{p} \quad \text { for } 1<p<2 .
$$


(d) If $\left|\left(f(X)-\pi_{n}(X)\right) / f(X)\right|<r<1$ almost everywhere on $E$, then

$$
\left\|\pi_{n}\right\|_{p}^{p}-\|f\|_{p}^{p} \leqq M_{5} \int_{E}|f|^{p-2}\left|f-\pi_{n}\right|^{2} d \mu \quad \text { for } p>1 .
$$

Proof. Identify the present $f$ and $\left(\pi_{n}-f\right)$ with $f$ and $g$ of Theorem 3. The sets $E_{1}$ and $E_{2}$, now denoted by $E_{1 n}$ and $E_{2 n}$, depend on $n$. By combining (a) and (b) of Theorem 3 and applying Theorem 4, we have

(5.1) $\left\|\pi_{n}\right\|_{p}^{p}-\|f\|_{p}^{p} \leqq A(p, r) \int_{E_{1 n}}|f|^{p-2}\left|f-\pi_{n}\right|^{2} d \mu+B(p, r) \int_{E_{2 n}}\left|f-\pi_{n}\right|^{p} d \mu$.

If $p \geqq 2$, by applying Hölder's inequality to the integral in the first term of the second member of (5.1) and noting that

$$
\int_{E_{2 n}}\left|f-\pi_{n}\right|^{p} d \mu \leqq\left[\int_{E_{2 n}}\left|f-\pi_{n}\right|^{p} d \mu\right]^{2 / p},
$$

we deduce that the right side of (5.1) is less than or equal to

$$
\max \left[A(p, r)\|f\|_{p}^{p-2} ; B(p, r)\right] \cdot\left\|f-\pi_{n}\right\|_{p,}^{2}
$$

which completes the proof of (a).

In (5.1) we factor sup $|f|^{p-2}$ from the first integral and, in case $\left|f-\pi_{n}\right|<1$ and $p \geqq 2$, substitute $\left|f-\pi_{n}\right|^{2}$ for $\left|f-\pi_{n}\right|^{p}$ in the second integral on the right without changing the sense of the inequality or the constant factors, thus obtaining (b) for $p \geqq 2$. If $1<p<2$ and $|(f-\pi) / f|<r<1$ on $E$, since $E_{2 n}$ is the null set, the result (b) is obtained from (5.1) by factoring $\sup |f|^{p-2}$ from the first integral on the right.

Noting $|f|^{p-2}\left|f-\pi_{n}\right|^{2}=\left|\left(f-\pi_{n}\right) / f\right|^{2-p}\left|f-\pi_{n}\right|^{p}$ and substituting into (5.1) in the case $1<p<2$, we obtain (c). In case (d), $E_{2 n}$ is the null set.

Corollary 5.1. In the inequalities of Theorem 5 , if $\|f\| \neq 0,\left\|\pi_{n}\right\|-\|f\|$ may be substituted for $\left\|\pi_{n}\right\|^{p}-\|f\|^{p}$.

Proof. If $\left\|\pi_{n}\right\|=\|f\|$, the result is trivial. Otherwise, the law of the mean applied to the function $X^{p}$ implies there exists $\xi_{n},\|f\|<\xi_{n}<\left\|\pi_{n}\right\|$, so that

$$
\left\|\pi_{n}\right\|^{p}-\|f\|^{p}=\left[\left\|\pi_{n}\right\|-\|f\|\right] p \xi_{n}^{p-1}>\left[\left\|\pi_{n}\right\|-\|f\|\right] p\|f\|^{p-1} .
$$

Theorem 6. Suppose $f$ is extremal for a convex subset $L_{*}$ of $L^{p}$ and suppose $\pi_{n}$ is a sequence in $L_{*}$ such that $\lim _{n \rightarrow \infty}\left\|\pi_{n}\right\|=\|f\| \neq 0$. Then for any $\psi_{n}\left(\in L_{*}\right)$ such that $\left\|\psi_{n}\right\| \leqq\left\|\pi_{n}\right\|$,

$$
\left\|f-\psi_{n}\right\|=O\left[\left(\left\|\pi_{n}\right\|-\|f\|\right)^{1 / r}\right], \quad n \rightarrow \infty,
$$

with $r=p$ for $p \geqq 2$ and $r=2$ for $1<p<2$. For $p \geqq 2$, if $L_{*}$ is admissible and if $\left\|f-\pi_{n}\right\|=O\left(\gamma_{n}\right)$ with $\lim _{n \rightarrow \infty} \gamma_{n}=0$, then 


$$
\left\|f-\psi_{n}\right\|=O\left(\gamma_{n}^{2 / p}\right), \quad n \rightarrow \infty .
$$

Proof. We apply an inequality on uniformly convex spaces proved by Hanner [6] for $p>1$. In the case $p \geqq 2$ a result of Clarkson [3] could be used. Since $\left\|\psi_{n}\right\|-\|f\| \leqq\left\|\pi_{n}\right\|-\|f\|$, it is sufficient to show

$$
\left\|f-\psi_{n}\right\|=O\left[\left(\left\|\psi_{n}\right\|-\|f\|\right)^{1 / r}\right] .
$$

Let $d=\|f\|$ and $\eta_{n}=\left\|\psi_{n}\right\|-\|f\|$. Then $\|f / d\|=1$ and $\left\|\psi_{n} /\left(d+\eta_{n}\right)\right\|=1$. Now

$$
\begin{aligned}
\left\|f / 2 d+\psi_{n} / 2\left(d+\eta_{n}\right)\right\| & \geqq\left\|\left(f+\psi_{n}\right) / 2 d\right\|-\left\|\psi_{n}\right\|\left[(1 / 2 d)-1 / 2\left(d+\eta_{n}\right)\right] \\
& \geqq 1-\eta_{n} / 2 d .
\end{aligned}
$$

By the theorem of Hanner $\left\|\psi_{n} /\left(d+\eta_{n}\right)-f / d\right\| \leqq \epsilon_{n}$, with $\epsilon_{n}=O\left(\eta_{n}^{1 / r}\right), n \rightarrow \infty$, with $r=2$ for $1<p<2$ and $r=p$ for $p \geqq 2$. Now

$$
\begin{aligned}
\left\|\psi_{n}-f\right\| & \leqq d\left[\left\|\psi_{n} /\left(d+\eta_{n}\right)-f / d\right\|+\| \psi_{n}\left(1 / d-1 /\left(d+\eta_{n}\right) \|\right]\right. \\
& \leqq d\left[\epsilon_{n}+\eta_{n} / d\right]=O\left(\eta_{n}^{1 / r}\right) .
\end{aligned}
$$

Combining Corollary 5.1, as applied to (a) of Theorem 5 and the result of the present theorem, we obtain (6.2).

For $2 \leqq p<\infty$, if $f$ is extremal for $L_{*}$, a convex subset of $L^{p}$, and $h$ is an arbitrary element of $L_{*}$, it can be proved easily from an inequality of Clarkson [3] that $\|h-f\|^{p} \leqq 2^{p-1}\left[\|h\|^{p}-\|f\|^{p}\right]$. For by Clarkson's inequality $\|h+f\|^{p}+\|h-f\|^{p} \leqq 2^{p-1}\left[\|h\|^{p}+\|f\|^{p}\right]$. Since $\|(h+f) / 2\| \geqq\|f\|$, we have $-\|h+f\|^{p} \leqq-2^{p}\|f\|^{p}$. Thus, $\|h-f\|^{p} \leqq 2^{p-1}\left[\|h\|^{p}-\|f\|^{p}\right]$. For $1<p<2$ there does not exist $M$ such that $\|h-f\|^{p} \leqq M\left[\|h\|^{p}-\|f\|^{p}\right]$. For if this inequality held it could be combined with the conclusion in Theorem 5 , $\left\|\pi_{n}\right\|^{p}-\|f\|^{p}<M_{3}\left\|f-\pi_{n}\right\|_{2}^{2}$, to obtain a degree of convergence stronger than maximal convergence in the case $f$ is analytic and bounded from zero on $\gamma$.

When $\left\{\epsilon_{n}\right\}$ is used henceforth in this paper, it is to be understood that for $n$ sufficiently large $0<\epsilon_{n+1} \leqq \epsilon_{n}<1$ and $\lim _{n \rightarrow \infty} \epsilon_{n}=0$.

THeOREm 7. Suppose $f$, such that $\|f\| \neq 0$, is extremal for an admissible convex subset $L_{*}$ of $L^{p}, p>1$. Suppose, for given $\left\{\epsilon_{n}\right\}$, there exists $\pi_{n}\left(\in L_{*}\right)$ such that $\left\|f-\pi_{n}\right\|_{p}<1$ and such that for some $M$

$$
\left|\left[f(X)-\pi_{n}(X)\right] / f(X)\right|<M \epsilon_{n} \text { when } X \in\left(E-E_{0}\right),
$$

where $E_{0}$ is a set of measure zero and is independent of $n$. If $\psi_{n} \in L_{*}$ and $\left\|\psi_{n}\right\| \leqq\left\|\pi_{n}\right\|$, then $\left\|f-\psi_{n}\right\|=O\left(\epsilon_{n}^{2 / r}\right), n \rightarrow \infty$, with $r=p$ for $p \geqq 2$ but $r=2$ for $1<p<2$.

Proof. Corollary 5.1, applied to (d) of Theorem 5, followed by (7.1) and Theorem 6, yields the result. 
Theorem 7 . Suppose $f$ is extremal for an admissible convex subset $L_{*}$ of $L_{p}, p>1$, and that for given $\left\{\epsilon_{n}\right\}$ there exists $\pi_{n}\left(\in L_{*}\right)$ such that

$$
\begin{aligned}
\left\|f-\pi_{n}\right\|_{p} & <1, \\
\left\|\left(f-\pi_{n}\right) / f\right\|_{2} & =O\left(\epsilon_{n}\right), \\
\left|\left(f-\pi_{n}\right) / f\right| & <r<1 \quad \text { on } E .
\end{aligned}
$$

Then, if $\|f\|_{p} \neq 0$ and if $f$ is bounded on $E$, the conclusion of Theorem 7 holds.

TheOREM 8. Suppose $f$ with $\|f\| \neq 0$ is extremal for an admissible convex subset $L_{*}$ of $L^{p}, p \geqq 2$, and that, for a given $\left\{\epsilon_{n}\right\}$, there exists $f_{n}\left(\in L_{*}\right)$ such that $\left\|f-f_{n}\right\|_{p}<1$, and that either

$$
\left\|f-f_{n}\right\|_{p}=O\left(\epsilon_{n}\right), \quad n \rightarrow \infty,
$$

or

(2) $f$ is bounded on $E,\left|f_{n}(X)-f(X)\right|<1$ almost everywhere on $E$, and

$$
\left\|f-f_{n}\right\|_{2}=O\left(\epsilon_{n}\right), \quad n \rightarrow \infty .
$$

If $\psi_{n}\left(\in L_{*}\right)$ is defined so that $\left\|\psi_{n}\right\|_{p} \leqq\left\|f_{n}\right\|_{p}$, then

$$
\left\|f-\psi_{n}\right\|_{p}=O\left(\epsilon_{n}^{2 / p}\right), \quad n \rightarrow \infty .
$$

Proof. Under the hypotheses (1) or (2), Corollary 5.1 as applied to (a) or (b) of Theorem 5 implies $\left\|f_{n}\right\|-\|f\|=O\left(\epsilon_{n}^{2}\right)$. Theorem 6 now gives the required result.

THEOREM 9. Suppose $f$ is extremal for an admissible subset $L_{*}$ of $L^{p}, p \geqq 2$. For given $\left\{\epsilon_{n}\right\}$ suppose $\pi_{n}\left(\in L_{*}\right)$ defined so that $\left\|f-\pi_{n}\right\|_{p}=O\left(\epsilon_{n}\right), n \rightarrow \infty$. Let $\psi_{n}$ be any sequence in $L_{*}$ such that $\left\|\psi_{n}\right\|_{p} \leqq\left\|\pi_{n}\right\|_{p}$. Then if $\left[|f|^{p-2}\right]^{-\beta}$ is integrable for some $\beta(>0)$, we have

$$
\int_{E}\left|f-\psi_{n}\right|^{2(1-a)} d \mu=O\left(\epsilon_{n}^{2(1-a)}\right) \quad \text { for } a=1 /(1+\beta) .
$$

Proof. We first derive (9.2), which holds for $p>1$ if $f$ is extremal for an admissible subset $L_{*}$ of $L^{p}$, and (9.3). Finally (9.5), (9.3), and (9.4) are combined to complete the proof.

We begin with the identity

$$
\begin{aligned}
\int|f|^{p-2}|f-g|^{2} d \mu= & \int|f|^{p-2} \bar{f}(f-g) d \mu-\int|f|^{p-2} f \bar{g} d \mu \\
& +\int|f|^{p-2}|g|^{2} d \mu
\end{aligned}
$$

which holds if $g \in L_{*}$. Theorem 4 implies $\int|f|^{p-2} \bar{f}(f-g) d \mu=0$, whence since $\int|f|^{p} d \mu$ is real, 


$$
\int|f|^{p-2} f \bar{g} d \mu=\int|f|^{p-2} \overline{f g} d \mu=\int|f|^{p} d \mu .
$$

Substitution of these results into (9.1) yields

$$
\int|f|^{p-2}|f-g|^{2} d \mu=\int|f|^{p-2}\left[|g|^{2}-|f|^{2}\right] d \mu .
$$

Hölder's inequality implies

$$
\int|f|^{p-2}|g|^{2} d \mu \leqq\left\{\int|f|^{p} d \mu\right\}^{(p-2) / p}\left\{\int|g|^{p} d \mu\right\}^{2 / p} .
$$

Since $f$ is extremal, the right member is not greater than $\int|g|^{p} d \mu$. This result combined with (9.2) implies that

$$
\int|f|^{p-2}|f-g|^{2} d \mu \leqq \int|g|^{p} d \mu-\int|f|^{p} d \mu .
$$

We note now that if $p>0$ and $r>0$, if $|f|^{p-2}|f-g|^{r}$ is integrable and if, for some $\beta(>0)$, also $\left[|f|^{p-2}\right]^{-\beta}$ is integrable, then

(9.4) $\int|f-g|^{r(1-a)} d \mu \leqq\left[\int d \mu /\left\{|f|^{p-2}\right\}^{(1-a) / a}\right]^{a} \cdot\left[\int|f|^{p-2}|f-g|^{r} d_{\mu}\right]^{1-a}$,

where $a=1 /(1+\beta)$, a result obtained by using $|f|^{p-2}$ as the weight function in an inequality previously proved $[19$, p. 105]. (The method of proof in which Hölder's inequality is applied holds for an arbitrary $L^{p}$-space.)

Now by hypothesis, $\left\|\pi_{n}\right\| \geqq\left\|\psi_{n}\right\| \geqq\|f\|$. Then

$$
\left\|\psi_{n}\right\|_{p}^{p}-\|f\|_{p}^{p}=O\left(\epsilon_{n}^{2}\right)
$$

is an immediate consequence of (a) of Theorem 5 .

Combining (9.5) with (9.3) and (9.4) when $g$ is replaced by $\psi_{n}$, we obtain the required result.

\section{Applications to analytic functions}

4. Convergence of sequences of functions extremal for line and surface integrals in the complex plane. The remainder of this paper is concerned with convergence, in particular, degree of convergence of the extremal polynomials to the corresponding extremal function in $H_{p}$ on certain subsets of the complex plane. We frequently use

Hypothesis H. Let $D$ be a closed limited Jordan region, with interior $C$, bounded by a Jordan curve $\gamma$ which, for the line integral case, is supposed analytic. Assume integration over $C$ for the surface integral, over $\gamma$ for the line integral. Define $L_{*}$ and $L_{n}$ as in Example B of the introduction. Suppose $F \in L^{p}$ 
defined on $E$, the set over which the integral is taken, and let $f^{*}$ and $f_{n}^{*}$ minimize $\|F-f\|_{p}, f \in L_{*}$, and $\left\|F-f_{n}\right\|_{p}, \dot{f}_{n} \in L_{n}$, respectively.

Before proceeding to consider degree of convergence under assumption of special behavior of the extremal function we consider convergence in the general case.

Theorem 10. Assume Hypothesis $\mathrm{H}$. Then $f_{n}^{*}(z)$ converges to $f^{*}(z)$ everywhere interior to $\gamma$, uniformly on any closed set interior to $\gamma$.

Proof. For the line integral case there exist $\left[23\right.$, p. 431] polynomials $P_{n}$ such that $\lim _{n \rightarrow \infty}\left\|f^{*}-P_{n}\right\|=0$, since $f^{*} \in H_{p}$. We define $p_{n}(z)$ as $G_{n}(z)$ $+P_{n}(z)$ with $G_{n}(z)$ the polynomial $[19, \$ 11.1]$ of degree $m-1$ such that $G_{n}\left(w_{k}\right)=u_{k}-P_{n}\left(w_{k}\right), k=1,2, \cdots, m$, and such that on $\gamma$ we have $\left|G_{n}(z)\right|$ $\leqq M \max _{k}\left|u_{k}-P_{n}\left(w_{k}\right)\right|$ if the points $w_{k}$ are distinct; a suitably modified inequality holds even if some $w_{k}$ are multiple. Then $p_{n} \in L_{n}$ and

$$
\begin{aligned}
\left\|f^{*}-p_{n}\right\| & \leqq\left\|f^{*}-P_{n}\right\|+\left\|P_{n}-p_{n}\right\|=\left\|f^{*}-P_{n}\right\|+\left\|G_{n}\right\| \\
& \leqq\left\|f^{*}-P_{n}\right\|+M^{\prime} \max \left|f^{*}\left(w_{k}\right)-P_{n}\left(w_{k}\right)\right| .
\end{aligned}
$$

Now $P_{n}(z)$ converges to $f^{*}(z)$ uniformly on any closed set interior to $\gamma[19$, $\$ 5.8]$ and, since the $w_{k}$ are interior to $\gamma$,

$$
\lim _{n \rightarrow \infty} \max \left|f^{*}\left(w_{k}\right)-P_{n}\left(w_{k}\right)\right|=0 .
$$

Thus,

$$
\lim _{n \rightarrow \infty}\left\|f^{*}-p_{n}\right\|=0
$$

Minkowski's inequality, applied again, yields

$$
\left\|F-p_{n}\right\|-\left\|F-f^{*}\right\| \leqq\left\|f^{*}-p_{n}\right\| .
$$

By Theorem 6, when $L_{*}^{\prime}=\left\{g \mid g=F-f, f \in L_{*}\right\}$ is taken as the $L_{*}$, $\pi_{n}=F-p_{n}$, as the $\pi_{n}$, and $F-f_{n}^{*}$ as the $\psi_{n}$ of that theorem with $F-f^{*}$ extremal for $L_{*}^{\prime}$, we have $\left\|f^{*}-f_{n}^{*}\right\|=O\left[\left(\left\|F-p_{n}\right\|-\left\|F-f^{*}\right\|\right)^{1 / r}\right]$. Combining this result with $(10.1)$, we obtain $\lim \left\|f^{*}-f_{n}^{*}\right\|=0$. It follows $[19$, $\$ 5.8]$ that $\lim f_{n}^{*}(z)=f^{*}(z)$ everywhere interior to $\gamma$, uniformly on any closed set interior to $\gamma$.

In the proof for the surface integral case $[5]$, or $[19$, p. 45] and $[19$, p. 109] are used.

Theorem 11. Suppose $L_{*}$ defined as in Example B (line integral over $|z|$ $=1)$. Let $P(z)$ be a polynomial of degree $m-1$ such that $P\left(w_{j}\right)=u_{j}, j=1$, $\cdots, m$, and $B(z)=I_{1}^{m}\left(z-w_{j}\right) /\left(1-\bar{w}_{j} z\right)$. Then, if $F \in L^{p}, f^{*}$ minimizes $\|F-f\|_{p}, f \in L_{*}$, if and only if $g^{*}(z)=\left(f^{*}(z)-P(z)\right) / B(z)$ minimizes $\left\|F_{1}-g\right\|_{p}, g \in H_{p}$ with $F_{1}(z)=(F(z)-P(z)) / B(z)$. 
Proof. $\|F-f\|=\|(F-P) / B-(f-P) / B\|$.

Corollary 11.1. $f^{*}$ minimizes $\|f\|_{p}, f \in L_{*}$, if and only if $g^{*}$ minimizes $\|(-P / B)-g\|_{p}, g \in H_{p}$.

The following theorem was proved in more general form by Rogosinski and Shapiro ([13] or [16]).

Theorem A. Suppose $F(z)$ is analytic for $t<|z|<1 / t, 0<t<1$. Then for some $t^{\prime}, t \leqq t^{\prime}<1$, the minimizing function $f^{*}$ for $\|F-f\|_{p}, f \in H_{p}$, norm on $|z|=1$, satisfies the following conditions. For $p=1, f^{*}(z)$ is analytic for $|z|<1 / t^{\prime}$. For $1<p<\infty, f^{*}(z)$ is analytic for $|z|<1 / t^{\prime}$ except possibly for isolated branch points in $1<|z|<1 / t^{\prime}$.

Corollary 11.2. The extremal function for $L_{*}$ defined as in Example B for the line integral over an analytic Jordan curve $\gamma$ is always analytic on $C+\gamma$.

Proof. The general case reduces to the unit circle case by mapping the interior of $\gamma$ onto the interior of $|w|=1$.

5. Maximal convergence of sequences of polynomials extremal for line and surface integrals in the complex plane. We now consider some cases where the sequence of polynomials $p_{n}^{*}(z)$, extremal for polynomials of degree $n$ contained in a closed convex subset $L_{*}$ of $L^{p}$, can be shown to converge maximally to the extremal function of $L_{*}$.

For a more complete definition of maximal convergence the reader is referred to [19, Chapter IV]. We let $D$ denote a closed limited set in the complex plane whose complement $K$ in the extended plane is connected; $D_{R}$ the equipotential locus in $K, G(x, y)=\log R>0$, and $\rho=\rho(f)$ the greatest number such that $f(z)$ is single-valued and analytic everywhere interior to $D_{\rho}$. A sequence of polynomials $\left\{p_{n}(z)\right\}$ with the property that for every $R<\rho$ there exists $M$ (which may depend on $R$ but is independent of $n$ and $z$ ) such that $\left|f(z)-p_{n}(z)\right| \leqq M / R^{n}, z \in D$, is said to converge maximally to $f(z)$ on $D$.

The case $F \in L_{*}, f^{*}=F$, is already known. For the sake of simplicity in certain theorems we exclude this case.

Theorem 12. Assume Hypothesis H. If $f^{*}$ is single-valued and analytic on $D$, we conclude the following. In case $p \geqq 2$, if $\left[\left(F-f^{*}\right)^{p-2}\right]^{-\beta}$ is integrable for some $\beta>0$, then the sequence $f_{n}^{*}(z)$ converges maximally to $f^{*}(z)$ on $D$. In case $1<p<2$, if $F-f^{*} \equiv Q_{q} \phi$ for some function $\phi$ continuous and nonvanishing on $E$ and $Q_{q}$ a polynomial, say of degree $q$, then the convergence is maximal on $D$.

Proof. We note that if $F$ is analytic on $\bar{E}$ (that is on $D$ in case of the surface integral, on $\gamma$ for the line integral case) and if $f^{*}$ is analytic on $D$ as in the line integral case (by Corollary 11.2), the requirements in the hypothe- 
sis for $\left(F-f^{*}\right)$ are satisfied, whence, according to the theorem, maximal convergence is implied. (See Corollary 12.1.)

Define $L_{*}^{\prime}$ as $\left\{g \mid g=F-f, f \in L_{*}\right\}$ and $L_{n}^{\prime}$ as $\left\{g_{n} \mid g_{n}=F-f_{n}, f_{n} \in L_{n}\right\}$. By Theorem 1 the extremal element $g^{*}$ of $L_{*}^{\prime}$ is just $\left(F-f^{*}\right)$ and $g_{n}^{*}$, extremal for $L_{n}^{\prime}$, is just $\left(F-f_{n}^{*}\right)$. We note that $g^{*}$ is not identically zero on $E$.

Case (i). $p \geqq 2$. Since $f^{*}$ is supposed analytic on $D$, the Tchebycheff polynomials $\pi_{n}(z)$ have the property that for any $R_{1}, R<R_{1}<\rho_{f^{*}}$, we have $\left|f^{*}(z)-\pi_{n}(z)\right|<M_{1}^{\prime} / R_{1}^{n}$ when $z \in D$. Such polynomials can be chosen so that $\pi_{n}\left(w_{i}\right)=f^{*}\left(w_{i}\right)=u_{i}$, as assigned. Thus, $\left\|g^{*}-\left(F-\pi_{n}\right)\right\|=O\left(1 / R_{1}^{n}\right)$. Since $\left(F-\pi_{n}\right) \in L_{n}^{\prime}$ and $g_{n}^{*}$ is extremal for $L_{n}^{\prime},\left\|g_{n}^{*}\right\| \leqq\left\|F-\pi_{n}\right\|$. Thus, the hypothesis for Theorem 9 is satisfied with $L_{*}^{\prime}$ and $L_{n}^{\prime}$ taken as the $L_{*}$ and $L_{n}$ of that theorem, $g^{*}$ and $g_{n}^{*}$ as the $f$ and $\psi_{n}$, and $\left\{1 / R_{1}^{n}\right\}$ as $\left\{\epsilon_{n}\right\}$. Hence, Theorem 9 yields

$$
\int\left|\left(F-f^{*}\right)-\left(F-f_{n}^{*}\right)\right|^{2(1-a)} d \mu=O\left(1 / R_{1}^{2 n(1-a)}\right)
$$

for some $a, 0<a<1$.

Since the integrand in the left member of (12.1) is just $\left|f^{*}-f_{n}^{*}\right|^{2(1-a)}$, we may apply a theorem previously proved $[19$, p.94] or $[19$, p.97], to obtain $\left|f^{*}(z)-f_{n}^{*}(z)\right|<M / R^{n}$ on $D$ for some $M$, as required.

Case (ii). $1<p \leqq 2$. This discussion holds for all $p>1$, but for $p>2$ the degree of convergence deduced is weaker than maximal convergence although it implies convergence throughout $C_{\rho 2 / p}$.

By hypothesis, $F-f^{*}=Q_{q} \phi$ with $Q_{q}$ a polynomial of degree $q$ and $\phi$ continuous and nonvanishing on $\bar{E}$. Since $f^{*}$ is analytic and single-valued on $D, f^{*}(z) / Q_{q}(z)=h(z)+q_{r}(z) / Q_{q}(z)$ with $h$ analytic on $D$ and $q_{r}$ a polynomial of degree not greater than $q$. Hence, the existence of polynomials $p_{n}(z)$, independent of $R$, of respective degrees $n[19$, p. 20] such that

$$
\left|h(z)-p_{n}(z)\right|<M_{1}^{\prime} / R_{1}^{n}
$$

when $z \in D$ implies $\left|f^{*}(z) / Q_{q}(z)-\left[p_{n}(z)+q_{r}(z) / Q_{q}(z)\right]\right|<M_{1}^{\prime} / R_{1}^{n}$ when $z \in D$ and the removable singularities are removed, that is,

$$
\left|\left\{f^{*}(z)-\left[p_{n}(z) Q_{q}(z)+q_{r}(z)\right]\right\} / Q_{q}(z)\right|<M_{1}^{\prime} R_{1}^{q} / R_{1}^{n+q} \text {. }
$$

The expression in square brackets is a polynomial $\pi_{n+q}$ of degree $n+q$. If $p_{n}(z)$ is required $[19$, p. 310$]$ to equal $h(z)$ at the points $w_{k}$, then $\pi_{n}\left(w_{k}\right)$ $=p_{n}\left(w_{k}\right) Q_{q}\left(w_{k}\right)+q_{r}\left(w_{k}\right)=f^{*}\left(w_{k}\right)$. Thus $\pi_{n}\left(w_{k}\right)=u_{k}$, that is, $\pi_{n} \in L_{n+q}$.

Since $\phi$ is continuous and does not vanish on $\bar{E}$, it follows that

$$
\left|\left[\left(F(z)-f^{*}(z)\right)-\left(F(z)-\pi_{n+q}(z)\right)\right] / Q_{q}(z) \phi(z)\right| \leqq M_{1}^{\prime \prime} / R_{1}^{n+q} \quad \text { on } E \text {. }
$$

Since $\left(F-\pi_{n+q}\right) \in L_{n+q}^{\prime}$, we have 


$$
\left\|g_{n+q}^{*}\right\| \leqq\left\|F-\pi_{n+q}\right\|, \quad g_{n+q}^{*} \text { extremal for } L_{n+q}^{\prime} .
$$

Now Theorem 7 yields

$$
\left\|g^{*}-g_{n+q}^{*}\right\|=O\left[\left(1 / R_{1}^{n+q}\right)^{(2, r)}\right], \quad g^{*} \text { extremal for } L_{*},
$$

that is,

$$
\left\|f^{*}-f_{n+q}^{*}\right\|=O\left[\left(1 / R_{1}^{n+q}\right)^{(2, r)}\right]
$$

with $r=2$ for $1<p<2$, but $r=p$ for $p \geqq 2$. As in Case (i), this implies the existence of $M_{2}^{\prime}$ such that

$$
\left|f^{*}(z)-f_{n+q}^{*}(z)\right| \leqq M_{2}^{\prime} / R^{2(n+q) / r} \text { for } z \text { on } D .
$$

Corollary 12.1. For $L_{*}$ and $L_{n}$ defined as in Example B for the line integral, if $F$ is analytic on $\gamma$ but $F \notin L_{*}$, then $f_{n}^{*}(z)$ converges maximally to $f^{*}(z)$, where $f^{*}$ minimizes $\|F-f\|_{p}, f \in L^{*}$, and $f_{n}^{*}$ minimizes $\left\|F-f_{n}\right\|, f_{n} \in L_{n}$.

6. Degree of convergence if the extremal function satisfies an integrated Lipschitz condition. In the following, classes of functions previously studied in [22] are used. If $\gamma$ is an analytic Jordan curve, a function $f(z)$ belongs to class $H(k, \alpha, p)$ on $\gamma(k$ a non-negative integer, $0<\alpha<1, p>1)$ provided $f^{(k)}(z)$ is of class $H_{p}(\gamma)$, and provided, with respect to arc-length $s$ on $\gamma$, $f^{(k)}(z)$ satisfies a $p$ th power integrated Lipschitz condition of order $\alpha$. The Zygmund class $Z(k, p)$ is similarly defined except that $f^{(k)}(z) \equiv \phi(s)$ is continuous and satisfies a condition of form

$$
\int_{\gamma}|\phi(s+h)+\phi(s-h)-2 \phi(s)|^{p} d s=O(h)
$$

instead of an integrated Lipschitz condition.

By Corollary $12.1,\left\{f_{n}^{*}(z)\right\}$ converges maximally to $f^{*}(z)$ if $F(z)$ is assumed to be analytic on $\gamma$. Weaker conclusions are obtained in Theorem 13 under the hypothesis $F \in L^{p}$.

Theorem 13. Assume Hypothesis $\mathrm{H}$, line integral case, $p \geqq 2$. Suppose $F \notin L_{*}$. If for some $k \geqq 0$, either $f^{*} \in H(k, \alpha, p), 0<\alpha<1$, or $f^{*} \in Z(k, p)$, $\alpha=1$, on $\gamma$ we conclude the following:

(a) $\mathcal{S}_{\gamma}\left|f^{*}-f_{n}^{*}\right|^{p}|d z|=O\left(1 / n^{2(k+\alpha)}\right)$ and if $2(k+\alpha)>1$

$$
\left|f^{*}(z)-f_{n}^{*}(z)\right| \leqq M / n^{[2(k+\alpha)-1 / p} \quad \text { on } C+\gamma ;
$$

(b) if $\left[\left|F-f^{*}\right|^{p-2}\right]^{-\beta}$ is integrable for some $\beta>0$, then

$$
\int_{\gamma}\left|f^{*}-f_{n}^{*}\right|^{2(1-a)}|d z|=O\left(1 / n^{2(k+\alpha)(1-a)}\right)
$$

for $a=1 /(1+\beta)$. If $2(1-a)(k+\alpha)>1$, then

$$
\left|f^{*}(z)-f_{n}^{*}(z)\right| \leqq M^{\prime} / n^{k+\alpha-1 / 2(1-a)} \text { on } C+\gamma .
$$


Proof. There exist polynomials $\pi_{n}(z)$ [22, Theorem 1] such that $\left\|f^{*}-\pi_{n}\right\|_{p}$ $=O\left(1 / n^{k+\alpha}\right)$. Such polynomials may be assumed to belong to $L_{*}$. (See the proof of Theorem 10.)

Proof of (a). Theorem '8, when applied to $L_{*}^{\prime}=\left\{g \mid g=F-f, f \in L_{*}\right\}$ with $f_{n}$ of Theorem 8 taken as $F-\pi_{n}$ and $\psi_{n}$ as $F-f_{n}^{*}$, implies the first part of (a). A theorem of Sewell [15, Theorem 4.2.1] yields the second part of (a).

Proof of (b). Theorem 9, applied to $L_{*}^{\prime}=\left\{g \mid g=F-f, f \in L_{*}\right\}$ with $\pi_{n}$ of Theorem 9 taken as $F-\pi_{n}$ and $\psi_{n}$ as $F-f_{n}^{*}$ implies the first part of (b); $[15$, p. 123] implies the second part of (b).

In the next theorem the surface integral is used. A degree of convergence of the minimizing polynomials to the minimizing function $f^{*}$ of $H_{p}^{\prime}$ is obtained provided $f^{*} \in H(k, \alpha, 2)$. When the norm notation is used here, the surface integral is to be understood.

Theorem 14. Assume Hypothesis $\mathrm{H}$ (surface integral case) with the additional requirement that the boundary $\gamma$ is an analytic Jordan curve.

Case (i). $p \geqq 2$. Suppose $F$ is bounded on $\gamma$ but $F \notin L_{*}$. If either $f^{*} \in$ $H(k, \alpha, 2)$ for $0<\alpha<1$ or $f^{*} \in Z(k, 2)$ for $\alpha=1$ on $\gamma$ and if $2(k+\alpha)>1$, then $\left\|f^{*}-f_{n}^{*}\right\|_{p}=O\left(1 / n^{[2(k+\alpha)+1] / p}\right)$ and $\left|f^{*}(z)-f_{n}^{*}(z)\right|<M / n^{[2(k+\alpha)+1] / p}$ on $D$.

Case (ii). $1<p<\infty$. Let $F(z) \equiv 0$. If $f^{*}=Q_{q} \phi$ for some polynomial $Q_{q}$, say of degree $q$, and $\phi$ bounded from zero on $D$ and of class $H(k, \alpha, 2), 0<\alpha<1$, or of class $Z(k, 2), \alpha=1$, on $\gamma$, then if $2(k+\alpha)>1$,

$$
\left\|f^{*}-f_{n}^{*}\right\|_{p}=O\left(1 / n^{(k+\alpha+1 / 2) 2 / r}\right),
$$

where $r=2$ for $1<p<2, r=p$ for $p \geqq 2$, and

$$
\left|f^{*}(z)-f_{n}^{*}(z)\right|<M^{\prime} / n^{(k+\alpha+1 / 2) 2 / r-2 / p}
$$

on $D$.

Proof of Case (i). There exists a function $\pi_{n}$ of $L_{n}$ such that $\left\|f^{*}-\pi_{n}\right\|_{2}$ $=\left\|\left(F-f^{*}\right)-\left(F-\pi_{n}\right)\right\|_{2}=O\left(1 / n^{k+\alpha+1 / 2}\right)$, by [21], [1a], or [24]. To verify that $\pi_{n}$ can be supposed to belong to $L_{*}$ see the proof of Theorem 10 . Then $\left[15\right.$, p. 137, Exercise 4.6.14] implies $\left|f^{*}(z)-\pi_{n}(z)\right|<M / n^{k+\alpha-1 / 2}$ on $D$. (There is a misprint in $\left[15\right.$, p. 137 , line 2 from the bottom]: $\left|f(z)-P_{n}(z)\right|$ should be raised to the $p$ th power.)

When Theorem 8 , (2) is applied with $F-f^{*}$ and $F-f_{n}^{*}$ taken respectively as $f^{*}$ and $f_{n}^{*}$, the theorem yields the required result for $\left\|f^{*}-f_{n}^{*}\right\|_{p}$. Application of [15, Exercise 4.6.14] completes the proof for Case (i).

Proof of Case (ii). $1<p<\infty$. We proceed to show Theorem $7^{\prime}$ can be applied. By [21], [ 1a], or [24], there exist polynomials $\pi_{n}$ of respective degree $n$ such that

$$
\left\|\phi-\pi_{n}\right\|_{2}=O\left(1 / n^{k+\alpha+1 / 2}\right) .
$$


These polynomials can be chosen so that $\pi_{n}\left(w_{k}\right)=u_{k} / Q_{q}\left(w_{k}\right)$. By $[15, \mathrm{Ex}-$ ercise 4.6.14], $\left|\phi(z)-\pi_{n}(z)\right|<M_{1} / n^{k+\alpha-1 / 2}$ on $D$, whence, since $f^{*}(z)$ $=\phi(z) Q_{q}(z)$ with $\phi(z)$ bounded from zero on $D,\left|\left[f^{*}(z)-\pi_{n}(z) Q_{q}(z)\right] / f^{*}(z)\right|$ $<M_{1} / n^{k+\alpha-1 / 2}<r<1$ on $D$. Clearly $\left\|f^{*}-\pi_{n} Q_{q}\right\|_{p}<1$ for large $n$. When $n>q, 1 / n^{k+\alpha+1 / 2}<2^{k+\alpha} /(n+q)^{k+\alpha+1 / 2}$, so (14.1) implies $\left\|\left(f^{*}-\pi_{n} Q_{q}\right) / f^{*}\right\|_{2}$ $=O\left(1 /(n+q)^{k+\alpha+1 / 2}\right)$. When the $\pi_{n} Q_{q}$ are taken as the polynomials of degree $n+q$ of Theorem $7^{\prime}$, that theorem yields $\left\|f^{*}-f_{n}^{*}\right\|_{p}=O\left(1 / n^{(k+\alpha+1 / 2) 2 / r}\right)$, whence $\left|f^{*}(z)-f_{n}^{*}(z)\right| \leqq M^{\prime} / n^{(k+\alpha+1 / 2) 2 / r-2 / p}$ on $D$, with $r=p$ for $p \geqq 2$ but $r=2$ for $1<p<2$.

Theorems 12-14 have immediate application to the specific problems mentioned in the introduction. A simple illustration is the (Bieberbach) case of a surface integral with $m=1, p=2$ and the condition $p_{n}(0)=1$; the extremal function $f(z)$ maps $C$ one-to-one onto a circular disc, and its continuity properties on $\gamma$ or $D_{\rho}$ yield by Theorems 12 and 14 immediate results on degree of convergence of the extremal polynomials. The application of Theorem 12, involving maximal convergence, is considered in [19, Chapter 11 , Theorem 7]; less general results than this application of Theorem 14 are given in [9a]. Likewise for the (Julia) problems of surface or line integrals with $m=2, p>1$, and the conditions $p_{n}(0)=0, p_{n}^{\prime}(0)=1$, the extremal function is $f(z)\left[f^{\prime}(z)\right]^{2 / p}$ or $f(z)\left[f^{\prime}(z)\right]^{1 / p}$, respectively, where $f(z)$ is the mapping function; continuity properties on $\gamma$ or $D_{\rho}$ of the latter yield by Theorems 12-14 immediate results on degree of convergence of the extremal polynomials. Maximal convergence has already been extablished in the case with $p=2$ for the line integral [ 19, Chapter 11, Theorem 9].

\section{BiBLIOGRAPHY}

1. L. V. Ahlfors, Variational methods in function theory, Lecture notes, Harvard University, Cambridge, Mass., 1953.

1a. S. J. Alper, Approximation of analytic functions in the mean over a region, Dokl. Akad. Nauk SSSR 136 (1961), 265-268; English transl., Soviet Math. Dokl. 2 (1961), 36-39.

2. L. Bieberbach, Zur Theorie und Praxis der konformen Abbildung, Rend. Circ. Mat. Palermo 38 (1914), 98-118.

3. J. A. Clarkson, Uniformly convex spaces, Trans. Amer. Math. Sor. 40 (1936), 396-414.

4. J. L. Doob, A minimum problem in the theory of analytic functions, Duke Math. J. 8 (1941), 413-424.

5. O. J. Farrell, On approximation to an analytic function by polynomials, Bull. Amer. Math. Soc. 40 (1934), 908-914.

5a. P. R. Garabedian, The classes $L_{p}$ and conformal mapping, Trans. Amer. Math. Soc. 69 (1950), 392-415.

6. O. Hanner, On the uniform convexity of $L^{p}$ and $l^{p}$, Ark. Mat. 3 (1956), 239-244.

7. G. Julia, Leçons sur la représentation conforme des aires simplement connexes, GauthierrVillars, Paris, 1931.

8. S. Kakeya, General mean modulus of analytic functions, Proc. Phys.-Math. Soc. Japan (3) 3 (1921), 48-58.

9. M. Keldyš and M. Lavrentieff, Sur la représentation conforme, C. R. Acad. Sci. URSS 1 (1935), 87-88. 
9a. M. Keldyš, Sur l'approximation en moyenne quadratique des fonctions analytiques, Mat. Sb. (N. S.) 5 (47) (1939), 391-401.

10. A. J. Macintyre and W. W. Rogosinski, Extremum problems in the theory of analytic functions, Acta Math. 82 (1950), 275-325.

11. J. Penez, Approximation by boundary values of analytic functions, Proc. Nat. Acad. Sci. U. S. A. 40 (1954), 240-243.

12. F. Riesz and M. Riesz, Über die Randwerte einer analytischen Funktion, C. R. 4th Congr. Math. Scand., 1916, pp. 27-44, Uppsala, 1920.

13. W. W. Rogosinski and H. S. Shapiro, On certain extremum problems for analytic functions, Acta Math. 90 (1953), 287-318.

14. P. C. Rosenbloom and S. E. Warschawski, A division problem for analytic functions, Seminars on analytic functions, Vol. 1, Institute for Advanced Study, Princeton, N. J., 1957.

15. W. E. Sewell, Degree of approximation by polynomials in the complex domain, Annals of Mathematics Studies No. 9, Princeton Univ. Press, Princeton, N. J., 1942.

16. H. S. Shapiro, Applications of normed linear spaces to function-theoretic extremal problems, Lectures on functions of a complex variable, pp. 399-404, Univ. of Michigan Press, Ann Arbor, 1955.

17. V. Smirnoff, Sur les formules de Cauchy et de Green et quelques problemes que s'y rattachent, Bull. Acad. Sci. USSR Ser. Math. 7 (1932), 337-371.

18. A. Spitzbart, Approximation in the sense of least pth powers with a single auxiliary condition of interpolation, Bull. Amer. Math. Soc. 52 (1946), 338-346.

19. J. L. Walsh, Interpolation and approximation by rational functions in the complex domain, Amer. Math. Soc. Colloq. Publ. Vol. 20, 2nd ed., Amer. Math. Soc., Providence, R. I., 1956. 20. N_ Note on invariance of degree of polynomial and trigonometric approximation under change of independent variable, Proc. Nat. Acad. Sci. U. S. A. 45 (1959), 1528-1533.

21. Note on least-square approximation to an analytic function by polynomials, as measured by a surface integral, Proc. Amer. Math. Soc. 10 (1959), 273-279.

22. J. L. Walsh and H. G. Russell, Integrated continuity conditions and degree of approximation by polynomials or by bounded analytic functions, Trans. Amer. Math. Soc. 92 (1959), 355-370. 23. , On simultaneous interpolation and approximation by functions analytic in a given

region, Trans. Amer. Math. Soc. 69 (1950), 416-439.

24. J. L. Walsh, Degree of polynomial approximation to an analytic function as measured by $a$ surface integral, Proc. Nat. Acad. Sci. U. S. A. 48 (1962), 26-32.

25. A. C. Zaanen, Linear analysis, North-Holland, Amsterdam, 1953.

HARVARD UNIVERSITY,

Cambridge, Massachusetts

Purdue University,

LAFA YETTE, INDIANA 